\title{
A linear Stark shift in dressed atoms as a signal to measure a nuclear anapole moment with a cold atom fountain or interferometer
}

\author{
Marie-Anne Bouchiat \\ Laboratoire Kastler Brossel, Département de Physique de l'Ecole Normale Supérieure, \\ 24 Rue Lhomond, F-75231 Paris Cedex 05, France
}

(Dated: November 3, 2006)

\begin{abstract}
We demonstrate theoretically the existence of a linear dc Stark shift of the individual substates of an alkali atom in its ground state, dressed by a circularly polarized laser field. It arises from the electroweak nuclear anapole moment violating $\mathrm{P}$ but not $\mathrm{T}$. It is characterized by the pseudoscalar $\xi \mathbf{k} \wedge \mathbf{E} \cdot \mathbf{B}$ involving the photon angular momentum and static electric and magnetic fields. We derive the relevant left-right asymmetry with its complete signature in a field configuration selected for a precision measurement with cold atom beams. The $3,3 \rightarrow 4,3$ Cs transition frequency shift amounts to $7 \mu \mathrm{Hz}$ for a laser power of $\approx 1 \mathrm{~kW}$ at $877 \mathrm{~nm}, \mathrm{E}=100 \mathrm{kV} / \mathrm{cm}$ and $\mathrm{B} \gtrsim 0.5 \mathrm{G}$.

PACS numbers: 32.80.Ys, 11.30.Er, 32.60.+i, 32.80.Pj
\end{abstract}

Atomic parity violation has built a bridge between atomic physics and both particle and nuclear physics [1]. The concept of a nuclear anapole moment 2] may be ascribed to toroidal currents [3]. Alternatively, it describes a chiral distribution of the atomic nucleus magnetization, i.e. a nuclear helimagnetism, resulting from the parity violating (PV) nuclear forces exerted inside a stable nucleus [4]. In a stationary atomic state, as a result of T-reversal invariance, only the nuclear spin dependent PV interaction discussed here contributes to observable PV effects, while in forbidden optical transitions the dominating nuclear spin-independent PV interaction, associated with the weak nuclear charge $Q_{W}$, makes the anapole effect difficult to extract.

Today, there exists only one empirical manifestation of this nuclear anapole moment, though an important nuclear property. It arises from PV measurements performed on the highly forbidden 6S-7S Cs transition, yielding electric dipole transition amplitudes, $E_{1}^{p v} \simeq 0.8 \times$ $10^{-11} e a_{0}$, of a slightly different magnitude $( \pm 2 \%)$ for the two hyperfine components $\Delta F= \pm 1$ [5]. The reported result presents significant discrepancies with different theoretical predictions and with other manifestations of the PV nuclear forces [3, 4]. On the experimental side, it is notoriously difficult to extract such a small fractional difference from measurements where the control of systematic effects represents a major task. In short, there is a clear need for new independent results both in atomic and nuclear physics. In this latter field, new high precision experiments are already underway, namely the measurement of the directional gamma-ray asymmetry in the $\mathrm{n}+\mathrm{p} \rightarrow \mathrm{d}+\gamma$ experiment and of the neutron spin rotation in helium [6]. We suggest here a new atomic physics approach using a frequency shift measurement in an atomic fountain or a matter-wave interferometer.

Like previous proposals [7, 8, 9, 10], this project is well suited to an atom in its ground state, where the effect of the dominant PV potential due to the weak charge cancels, but it presents important additional advantages.
First, instead of relying on the left-right asymmetry in a transition rate, it is based, as in [10], on an energy shift of the atomic states, and its measurement takes advantage of the methods developed for the primary ${ }^{133} \mathrm{Cs}$ standard. This experimental domain and that of atomic interferometry [11, 12] have now reached an impressive, continuously improving, level of accuracy. Nowadays, beyond their metrological interest, cold atom fountains and interferometers are becoming undisputable tools, leading to a wide variety of high precision measurements of fundamental interest, such as a lower limit on the time variation of the fine structure constant 13], new tests of Lorentz invariance (LI) 14], possibly tighter constraints on the electron EDM 15, 16 and new tests of the Einstein Equivalence Principle [13]. Second, this anapole shift is both a light shift and a dc linear Stark shift of the $\left|F, m_{F}\right\rangle$ ground state sublevels, violating $\mathrm{P}$ but not $\mathrm{T}$. It is quadratic in the parity conserving (PC) atom radiation field interaction, and linear in the static interaction of the nuclear anapole moment with a dc Stark field. Its physical origin is then totally different from the PV light-shift involved in a project for a measurement of $Q_{W}$ on a single trapped $\mathrm{Ba}^{+}$ion [17. In the present project, one must apply both a static electric field and a strong laser field, but its phase is irrelevant. So it is not necessary to localize the atoms inside a cavity unlike the case of previous proposals [8, 9].

The nuclear spin-dependent PV interaction is responsible not only for a transition electric dipole but also for a permanent one in an alkali atom ground state, involving the operator $\mathbf{s} \wedge \mathbf{I}$, s and $\mathbf{I}$ being the spin of the electron and the nucleus respectively. Thus, in an applied static electric field $\mathbf{E}$, the nuclear anapole moment gives rise to the time-independent $\mathrm{P}$-odd, T-even, interaction $V_{\text {ana }}=d_{I} \mathbf{E} \cdot \mathbf{s} \wedge \vec{I} \equiv-d_{I} \frac{i}{2}\left[F^{2}, \mathbf{s} \cdot \mathbf{E}\right]$, where $\mathbf{F}=\mathbf{s}+\mathbf{I}$. This identity results from properties of the Pauli matrices, $\vec{\sigma}=2 \mathrm{~s}$. The commutator appearing in the last expression shows directly that $V_{\text {ana }}$ has matrix elements only between states belonging to different $\mathrm{F}$ multiplets. 
From previously published data [5], the electric dipole moment $d_{I}$ for the Cs ground state can be estimated to $d_{I} \simeq 2.36(40) \times 10^{-13}|e| a_{0}[10]$.

We assume that the atom in its ground state is also perturbed by the electric field of a laser beam of frequency $\omega$ detuned from an allowed transition of frequency $\omega_{0}=\omega-\delta$. Supposing the beam circularly polarized, we write the associated electric classical field $\mathbf{E}_{c}(\mathbf{r}, t)$ :

$$
\mathbf{E}_{c}(\mathbf{r}, t)=\frac{1}{\sqrt{2}} \mathcal{E}(\mathbf{e}(\xi) \exp (-i \omega t+i \mathbf{k} \cdot \mathbf{r})+\text { c.c. }),
$$

with $\mathbf{e}(\xi)=\frac{1}{\sqrt{2}}\left(\mathbf{e}_{1}+i \xi \mathbf{e}_{2}\right), \mathbf{e}_{i}$ being two orthogonal unit real vectors normal to the wave vector $\mathbf{k}$ of the laser beam and $\xi= \pm 1$ defining the helicity. The energy density of the beam $\epsilon_{0} \mathbf{E}_{c}(\mathbf{r}, t)^{2}$ is then given by $\epsilon_{0} \mathcal{E}^{2}$. We write the quantized form of the radiation electric field, assuming that only the laser mode $\mathbf{e}(\xi) \exp (-i \omega t+i \mathbf{k} \cdot \mathbf{r})$ is involved in the radiative transition. The associated annihilation (creation) operator $a\left(a^{\dagger}\right)$ is normalized in such a way that the one photon state obtained by applying $a^{\dagger}$ upon the vacuum state $|0\rangle$ has its energy $\hbar \omega$ localized inside a volume $V$. If the laser beam is described by a normalized $N$ photon state $|N\rangle$, its energy density is: $\epsilon_{0} \mathcal{E}^{2}=N \hbar \omega / V$.

The combined atom-field system is described in terms of its eigenstates $|i\rangle|N\rangle$, where the first ket refers to the atomic states and the second to the radiation field states. The coupling of the ground state to the nearly resonant excited state via virtual photon absorption and emission gives the dominant contribution to the ac Stark shift, or light-shift, of the $6 S_{1 / 2}$ substates. The hamiltonian $V_{\text {rad }}=-\sqrt{\frac{\hbar \omega}{2 \epsilon_{0} V}}(a \mathbf{e}(\xi) \cdot \mathbf{D}+$ h.c. $)$ describes the atomfield dipolar coupling. Its matrix elements between the initial state, $|i\rangle=\left|n S_{1 / 2}\right\rangle|N\rangle$, and the first excited state $|f\rangle=\left|n^{\prime} P_{J}\right\rangle|N-1\rangle$ read:

$$
\left\langle f\left|V_{\text {rad }}\right| i\right\rangle=-\frac{1}{\sqrt{2}} \mathcal{E}\left\langle n^{\prime} P_{J}|\mathbf{e}(\xi) \cdot \mathbf{D}| n S_{1 / 2}\right\rangle .
$$

We write the lowest-order non-zero modification to the ground state energy involving both perturbations $V_{a n a}$ and $V_{\text {rad }}$. It is linear in $V_{a n a}$ and quadratic in $V_{\text {rad }}$ :

$$
\begin{gathered}
E_{\alpha}^{(3)}=\left\langle\alpha\left|V_{\text {ana }} R V_{\text {rad }} R V_{\text {rad }}\right| \alpha\right\rangle+ \\
\left\langle\alpha\left|V_{\text {rad }} R V_{\text {ana }} R V_{\text {rad }}\right| \alpha\right\rangle+\left\langle\alpha\left|V_{\text {rad }} R V_{\text {rad }} R V_{\text {ana }}\right| \alpha\right\rangle .
\end{gathered}
$$

In this general expression, the hyperfine and the Zeeman structures are now included in the description of the atom-field states: $|\alpha\rangle=\left|n S_{1 / 2} ; F, m_{F}\right\rangle|N\rangle$, the resolvent operator $R=\left(E_{n}-H\right)^{-1}$ involves the unperturbed hamiltonian $H$ and the ground state energies $E_{n}$ of the combined atom-field system. As it will become clear later on, the condition $|\Delta W / \hbar \delta| \ll 1$ has to be satisfied in a realistic experiment. Therefore, the second term in Eq.(3) can be neglected because its energy denominator $|\hbar \delta|^{2}$ is much larger than those of the two other terms, $\Delta W|\hbar \delta|$.

$$
E_{\alpha}^{(3)}=\frac{\left(F-F^{\prime}\right)}{\Delta W}\left\langle\alpha\left|V_{\text {ana }}\right| \alpha^{\prime}\right\rangle\left\langle\alpha^{\prime}\left|V_{\text {rad }} R V_{\text {rad }}\right| \alpha\right\rangle+\text { h.c. }
$$

Here the state $\left|\alpha^{\prime}\right\rangle$ differs from $|\alpha\rangle$ just by the change of $F, m_{F}$ into $F^{\prime} \neq F, m_{F}^{\prime}$. The last factor is the secondorder modification to the ground state energy arising from its coupling with the radiation field. First, we limit ourselves to the effect of the laser detuning with respect to the single $P_{1 / 2}$ excited state. In this case, $V_{\text {rad }}$ operates between $S_{1 / 2}$ and $P_{1 / 2}$, i.e. two electronic states of angular momentum $J=\frac{1}{2}$, and the atomic part of the operator involved can be simplified to $V_{\text {rad }}=\hbar \Omega_{1} \vec{\sigma} \cdot \hat{\epsilon}$, where $\Omega_{1}=d \mathcal{E} / \hbar \sqrt{2}$ is the Rabi frequency, and $d$ the $\Delta m_{s}=0$ electric dipole matrix element. The operator $\mathrm{R}$ can be replaced by the projection operator $\mathcal{P}_{P_{1 / 2}}=\sum_{m}\left|P_{1 / 2}, m\right\rangle\left\langle P_{1 / 2}, m\right|$ divided by $\hbar \delta$. A change of $F$ into $F^{\prime} \neq F$ can be produced only by the vector part of the tensor operator $V_{\text {rad }} \mathcal{P}_{P_{1 / 2}} V_{\text {rad }}$, which is just $\hbar \Omega_{1}^{2} \vec{\sigma} \cdot \xi \mathbf{k}$, if we introduce the angular momentum of the photons $\xi \mathbf{k}=i\left(\hat{\epsilon} \wedge \hat{\epsilon}^{*}\right) \hbar$. Then, we can substitute the expressions of $V_{a n a}$ and $V_{\text {rad }}$ into Eq.(4), to obtain the anapole energy shift of a $\left|F, m_{F}\right\rangle$ substate:

$$
\begin{gathered}
h \Delta \nu_{F, m_{F}}^{a n a}=\frac{d_{I} \Omega_{1}^{2}}{\Delta W \delta} \times \\
\sum_{F^{\prime}, m_{F}^{\prime}}\left(F^{\prime}-F\right)\left\langle F, m_{F}|\mathbf{E} \cdot \vec{\sigma} \wedge \mathbf{I}| F^{\prime}, m_{F}^{\prime}\right\rangle\left\langle F^{\prime}, m_{F}^{\prime}|\vec{\sigma} \cdot \xi \mathbf{k}| F, m_{F}\right\rangle .
\end{gathered}
$$

Formally, the summation over $F^{\prime}$ can be extended to all states, although only the states $F^{\prime} \neq F$ do contribute. By using a closure relation, we obtain the compact expression $\left\langle F, m_{F}|\mathbf{E} \cdot \xi \mathbf{k} \wedge \mathbf{I}| F, m_{F}\right\rangle$. Taking as quantization axis the direction defined by the applied magnetic field $\mathbf{B}$, we arrive at the final expression for the anapole shift:

$$
h \Delta \nu_{F, m_{F}}^{a n a}=2(F-I)(\xi \hat{k} \wedge \hat{E} \cdot \hat{B}) \frac{d_{I} E \hbar \Omega_{1}^{2}}{\Delta W \delta}\left\langle F, m_{F}\left|I_{z}\right| F, m_{F}\right\rangle,
$$

where $\hat{B}, \hat{k}$ and $\hat{E}$ are respectively unit vectors parallel to $\mathbf{B}, \mathbf{k}$ and $\mathbf{E}$. The $I_{z}$ matrix elements take the form $\lambda_{F} m_{F}$, in Cs $\lambda_{F}=\frac{7}{8}$ and $\frac{9}{8}$ for $\mathrm{F}=4$ and 3 respectively. We note that shifts of opposite signs are predicted for the two $\left|F=I \pm \frac{1}{2}, m_{F}\right\rangle$ states, hence both contribute constructively to the frequency shift of the $|\Delta F|=1, \Delta m_{F}=0$ transitions which, thus, scales as $2 m_{F}$. If $\mathbf{B}$ defines the quantization axis (see below), then, its magnitude has no effect on the anapole shift at the second order in $V_{\text {rad }}$ where Eq.6 is only valid.

The presence of the pseudoscalar quantity $\xi \hat{k} \wedge \hat{E} \cdot \hat{B}=$ $\chi$ is the mark of parity violation [18]. If the two fields and $\xi \mathbf{k}$ form a rectangular trihedron, its chirality $\chi$ takes the value \pm 1 . Reversals of $\xi, \mathbf{E}, \mathbf{B}$ and $m_{F}$ would be the indispensable operations used to identify the anapole frequency shift. They are all the more helpful since, without special care, this shift may appear superposed on a 

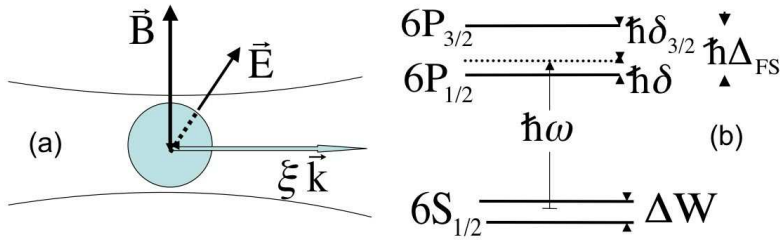

FIG. 1: (a) Scheme of the interaction region located close to the apex of the cold atom fountain. (b) Schematics of the relevant Cs atomic levels and laser detunings for $\delta_{3 / 2} / \delta<0$.

larger PC light-shift associated with the scalar part of the operator $V_{\text {rad }} \mathcal{P}_{P_{1 / 2}} V_{\text {rad }}$. Due to the slight detuning difference between the two $\mathrm{F}$ states, the scalar light-shift of a $\left|F, m_{F}\right\rangle$ state depends on $F$ to first order in $\Delta W / \hbar \delta$. Thus, the well known shift of the hyperfine splitting is $-\frac{\Delta W \Omega_{1}^{2}}{h \delta^{2}}$, whatever the sign of $\delta$. As for the contribution of the $P_{3 / 2}$ state, when its frequency detuning $\delta_{3 / 2}$ is comparable to $\delta$, it always reinforces that of $P_{1 / 2}$.

Let us, now, consider the relative contributions to the anapole and the PC light-shifts coming from the vector parts of the operator $V_{r a d} R V_{r a d}$ associated with both $P_{J}$ states. The basic property of the projection operators implying $\mathcal{P}_{P_{3 / 2}}=\mathbf{1}-\mathcal{P}_{P_{1 / 2}}$, where $\mathbf{1}$ is the unit operator, allows us to conclude that they are just opposite. Once weighted by the energy denominators, $\hbar \delta_{1 / 2} \equiv \hbar \delta$ and $\hbar \delta_{3 / 2}=-\rho \hbar \delta$, we see that, provided that the detunings be of opposite signs ( $\rho>0$, Fig.1b), both contributions add together and reinforce both the anapole shift (Eqs.(4) to (6)) and the vector PC light-shift $\xi \frac{\hbar \Omega_{1}^{2}}{\delta}$ by the factor $(1+\rho) / \rho$. (Note that the parameter $\rho$ is simply related to $\delta$ by $(1+\rho) \delta=\Delta_{F S}$, the fine structure interval and appears just for writing convenience). As shown in [19], the light shift induced within the $6 S_{1 / 2}$ sublevels by a circularly polarized beam can be described with the help of a fictitious magnetic field $\mathbf{B}_{l s}=\xi \mathbf{k} \frac{\hbar \Omega_{1}^{2}}{|\delta| \mu_{B}} \frac{1+\rho}{\rho}$. This provides a useful bench-mark to compare Zeeman and light shifts. As an example, if $\mathbf{k} \cdot \mathbf{B}=0$, the tilt of the quantization axis induced by $\mathbf{B}_{l s}$ leads a correction to the anapole shift of Eq.6 which reads to lowest order: $\frac{\delta \Delta \nu^{a n a}}{\Delta \nu^{a n a}} \approx-\frac{1}{2}\left(\frac{B_{l s}}{B}\right)^{2}$ for $B \gtrsim B_{l s}$. For $\mathbf{k} \cdot \mathbf{B}=0$ the $\xi$-dependence of the hyperfine splitting also cancels.

Before discussing the anapole shift magnitude, let us indicate how the measurement might be conducted in a fountain clock type experiment. A ball of cold atoms in an $\left|F, m_{F}\right\rangle$ state is launched vertically along the applied magnetic field. It passes through a microwave cavity and gets prepared in a coherent superposition of the $F, m_{F}$ and $F^{\prime}, m_{F}^{\prime}=m_{F}$ states described by the wave function $|\psi\rangle=\left(\left|F, m_{F}\right\rangle|N\rangle+\left|F^{\prime}, m_{F}\right\rangle|N\rangle\right) / \sqrt{2}$. After rising a distance $L$ above the cavity centre, the atoms reach the interaction region, located close to the apex of their trajectory. The dc field $\mathbf{E}$, the polarized laser beam $\xi \mathbf{k}$, and B form a rectangular trihedron (Fig.1a). Due to reversal of the atomic velocity, the very small motional magnetic field cancels. The beam waist should be chosen larger or equal to the atomic ball radius, $\sim 5 \mathrm{~mm}$. The atoms falling back reach the detection region. As usual in a fountain clock the measurement relies on the phase shift of the atomic superposition accumulated during the total time $\tau_{f}$ elapsed since its preparation, $\int \phi(t) d t$. Since the interaction lasts for a limited duration $\tau_{i}$, we expect the variation of the phase shift for the interaction on and off $\left(\Delta \nu_{F, m_{F}}^{a n a}-\Delta \nu_{F^{\prime}, m_{F}}^{a n a}\right)$, to be reduced by the factor $\tau_{i} / \tau_{f} \simeq \sqrt{2 w / L}$, typically 0.18 .

Up to now, $\Omega_{1}$ has appeared as a free parameter. However, it must be kept in mind that it cannot be chosen arbitrarily large : from the coupling induced between the ground and excited states by the radiation field results an instability of the atomic sample crossing the interaction region, hence a signal loss. The decay rate acquired by the $6 \mathrm{~S}$ atoms is easily computed by perturbation theory in the limit $\Gamma_{P J} \ll \Omega_{1}, \Gamma_{S}=\frac{\Omega_{1}^{2}}{\delta^{2}} \Gamma_{P_{1 / 2}} \kappa$, with $\kappa=1+\frac{R_{R a b i}^{2}}{\rho^{2}} \Gamma_{P_{3 / 2}} / \Gamma_{P_{1 / 2}}$, where $R_{R a b i}$ is the ratio of the Rabi frequencies for $P_{3 / 2}$ and $P_{1 / 2}$. Using numerical values from [20], $R_{R a b i}^{2}=1.98$ and $\kappa=1+2.27 / \rho^{2}$ for Cs. The condition to avoid excessive signal loss is $\Gamma_{S} \tau_{i} \leq 1$. Assuming this limit just reached, the anapole shift of the $F, m_{F} \rightarrow F+1, m_{F}$ transition angular frequency becomes:

$$
\begin{aligned}
& 2 \pi \Delta \nu_{m_{F}, m_{F}}^{a n a}=2 \chi m_{F} \frac{1+\rho}{\rho} \frac{d_{I} E}{\Delta W} \frac{\Omega_{1}^{2}}{\delta} \frac{\tau_{i}}{\tau_{f}} \\
& =2 \chi m_{F} \frac{(1+\rho)}{\rho} \frac{d_{I} E}{\Delta W \tau_{f}} \frac{\delta}{\kappa \Gamma_{P_{1 / 2}}}, \text { for } \Omega_{1}^{2}=\frac{\delta^{2}}{\kappa \Gamma_{P_{1 / 2} \tau_{i}}}(8)
\end{aligned}
$$

Eq.(8) shows that, due to the constraint imposed on the laser intensity, it is important to choose $\hbar \delta / \Delta W \gg 1$, as announced earlier. Using $\rho$ as the sole free parameter, it is easily found that $\Delta \nu_{m_{F}, m_{F}}^{a n a}$ as a function of $\rho$ presents a maximum for $\rho=\sqrt{2.27}$, leading to $\kappa=2$.

Inserting in Eq.(8), $E=100 \mathrm{kV} / \mathrm{cm}, d_{I} E / h=30 \mathrm{mHz}$, $\delta / 2 \pi=6.65 \mathrm{THz}, \Delta W / h=9.2 \mathrm{GHz}, \tau_{f}=0.5 \mathrm{~s}, \tau_{i} / \tau_{f}=$ $0.18, \Gamma_{P_{1 / 2}}^{-1}=35 \mathrm{~ns}$ and $\rho=1.50$, we obtain for Cs:

$$
\text { and } \quad \begin{aligned}
\Delta \nu_{3,3}^{a n a} & =7.6 \mu \mathrm{Hz}, \\
\Omega_{1} / 2 \pi & =2.9 \mathrm{GHz}, \quad B_{l s}=0.24 \mathrm{G} .
\end{aligned}
$$

For a beam radius of $5 \mathrm{~mm}$, the laser power should be $\approx 1 \mathrm{~kW}$. This can be achieved inside a Fabry-Perot cavity with a reasonable input power tuned at $877 \mathrm{~nm}$ [21]. The condition of adiabatic passage is largely satisfied by the atom-radiation field interaction. One might object that the magnitude chosen for $E$ is large. However, still larger fields $(450 \mathrm{kV} / \mathrm{cm})$ have been achieved before, for quadratic Stark effect measurements with heated glass electrodes 22]. The quadratic differential dc Stark shift [23], $\Delta \nu^{d c} \simeq 10 \mathrm{kHz}$, and the smaller scalar light-shift, $\Delta \nu^{l s}=-\left(1+\frac{R_{R a b i}^{2}}{\rho^{2}}\right) \frac{\Delta W \Omega_{1}^{2}}{h \delta^{2}} \frac{\tau_{i}}{\tau_{f}}$, could be eliminated via the fastest parameter reversals among the set $m_{F}, \xi, E, B$. 


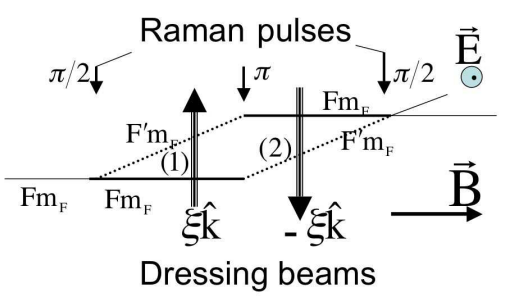

FIG. 2: Scheme of the interferometer. Both paths cross two dressing beams having opposite photon angular momenta, $\xi \mathbf{k}$.

It is clearly of interest to perform a calibration of the anapole shift allowing for a determination of the basic parameter $d_{I} E$ free of the uncertainties coming from the laser power and the exact geometry. We suggest using the PC scalar light-shift obtained by offsetting sequentially the laser detuning by a fractional amount $\pm \eta$ from one side of its best value to the other. In this case, $\Delta \nu_{\eta-o d d}^{l s}=$ $\Omega_{1}^{2} \frac{\tau_{i}}{\tau_{f}} \times 0.84 \frac{\Delta W}{h \delta^{2}} \eta$ is the light-shift odd in the $\eta$ reversal for cesium, while $\Delta \nu^{a n a}$, measured at its optimum, is not affected to first order in $\eta$. In the ratio,

$$
\mathcal{R}(\chi)=\frac{\Delta \nu_{3,3}^{a n a}}{\Delta \nu_{\eta-\text { odd }}^{l s}}=12 \cdot \chi \frac{d_{I} E}{\Delta W} \frac{\hbar \delta}{\Delta W} \eta^{-1},
$$

which bears the PV signature $\chi, \Omega_{1}^{2} \tau_{i} / \tau_{f}$ is eliminated and the only unknown quantity is just that we need, $d_{I} E / \Delta W$. The intensity-independent left-right asymmetry $\mathcal{R}(+1)-\mathcal{R}(-1)$ affecting the hyperfine transition frequency is definitely the quantity to be measured. It amounts to $\approx 1.2 \times 10^{-3}$ for $\eta=0.45 \times 10^{-4}$ (laser frequency excursions of $3 \mathrm{MHz}$ ). The precision will depend on the magnitude of $\eta$, and the frequencies of the parameter reversals adjusted for limiting the effect of harmful drifts. The most severe of these are likely to be the $\mathbf{B}$ drifts. Since the $3,0 \rightarrow 4,0$ transition is insensitive to the anapole shift, measurements should be performed on a first-order Zeeman shifted transition, as in the case of the LI tests [14]. A dual Cs-Rb fountain, already used for a fundamental physics test [13], could be very helpful, since frequency comparisons should reduce considerably the magnetic noise.

Another approach would be to use a cold atom interferometer. In the case where the wave packets are separated and recombined by using a $\pi / 2-\pi-\pi / 2$ sequence of Raman pulses [12], both paths can be made to cross two interaction regions having chiralities of opposite signs (Fig.2). In the phase difference $\Phi$ between the two paths, it is easily verified that the Zeeman shift cancels while the anapole shift contribution doubles. The same property holds for the calibration signal if $\eta$ is applied with opposite signs on the two dressing beams. An estimate gives $\Phi \approx 10 \mu \mathrm{rad}$ for $\tau_{f}=100 \mathrm{~ms}, \tau_{i} / \tau_{f}=0.2, \Omega_{1}^{2}$ cut off by 2 for keeping $\Gamma_{S}$ as all other parameters the same. Since no absolute measurements is required, this very specific phase shift looks accessible in the present state of the art.
In conclusion, we have shown the existence of a linear atomic Stark shift. with a chiral character providing a signal for the nuclear anapole moment. We have exhibited a concrete example where several powerful techniques developed recently in atomic physics for high precision frequency determinations appear as highly valuable tools for precise atomic PV measurements. The effect involved presents a certain similarity with the PV NMR frequency shifts expected in enantiomer molecules 24]. The chiral configuration defined by $\xi \mathbf{k}, \mathbf{E}, \mathbf{B}$, is playing here the role of the chiral arrangement of the atoms inside the molecule, but with the advantage of providing numerous reversals. Owing to the absence of any contribution due to the weak nuclear charge, one could thus obtain a rather direct measurement of a PV static property of the nucleus. The method could be extended to other atoms, in particular to strings of (possibly radioactive) isotopes and would provide valuable information about nuclear parity violation.

The author is grateful to C. Bouchiat, J. Guéna and M. D. Plimmer for helpful remarks. Laboratoire Kastler Brossel is a Unité de Recherche de l'Ecole Normale Supérieure et de l'Université Pierre et Marie Curie, associée au CNRS (UMR 8552).

[1] M. A. Bouchiat and C. Bouchiat, J. Phys. France, 35, 899-927 (1974) and Rep. Prog. Phys. 60, 1351 (1997).

[2] Y. B. Zel'dovich, Sov. Phys. JETP 9, (1959).

[3] V.V. Flambaum and I.B. Khriplovich, Sov. Phys. JETP 52, 835 (1980); J.S.M. Ginges and V.V. Flambaum, Phys. Rep. 397, 63 (2004).

[4] C. Bouchiat and C.A. Piketty, Z. Phys. C 49, 91 (1991).

[5] C. S. Wood et al., Science, 275, 1759 (1997).

[6] J. D. Bowman et al., Proceedings of the PAVI06 workshop, Eur. Phys. J. A in press; W. M. Snow et al., ibid.

[7] C. E. Loving et al., J. Phys. B 10, 2755 (1977).

[8] V. F. Ezhov et al., Tech. Phys. Lett 30, 917 (2004).

[9] E. Gomez et al., arXiv: physics/ 0412124 (2004).

[10] M. A. Bouchiat et al., Eur. Phys. J. D 15, 5 (2001).

[11] Ch. Bordé, Phys. Lett. A 140, 10 (1989).

[12] M. Kasevich and S. Chu, Phys. Rev. Lett. 67, 181 (1991).

[13] H. Marion et al., Phys. Rev. Lett. 90, 150801 (2003).

[14] P. Wolf et al., Phys. Rev. Lett. 96, 060801 (2006).

[15] J. M. Amini et al., arXiv: physics/0602011

[16] J. J. Hudson et al., Phys. Rev. Lett. 89023003 (2002).

[17] E. N. Fortson, Phys. Rev. Lett. 70, 2383 (1993).

[18] For a linearly polarized laser beam, the pseudoscalar involved is $(\hat{\epsilon} \cdot \hat{B})(\hat{\epsilon} \cdot \hat{B} \wedge \hat{E})$, but the effect cancels unless the $P_{J}$ state hyperfine interaction is taken into account. This reduces its magnitude by a factor $E_{h f} / \delta \approx 10^{-4}$.

[19] C. Cohen-Tannoudji et al., Phys. Rev. A 5, 968 (1972).

[20] C. Amiot et al., Phys. Rev. A 58, 1087 (1998).

[21] Choosing $\rho=R_{\text {Rabi }}^{2}$ cancels the radiative potential, and its possible perturbative effect on the atomic motion, at the price of a reduction of $\Delta \nu^{a n a}$ of only $4 \%$.

[22] H. Gould, Phys. Rev. A 14, 922 (1976).

[23] E. Simon et al., Phys. Rev. A 57, 436 (1998).

[24] A. L. Barra et al., Eur. Phys. Lett. 5, 217 (1988). 